J. Austral. Math. Soc. 19 (Series A) (1975), 247-251.

\title{
LOCALLY COMPACT TIGHT RIESZ GROUPS
}

\author{
ANDREW WIRTH
}

(Received 12 May 1972)

Communicated by J. B. Miller

\section{Introduction}

It is known that a strongly archimedean locally compact tight Riesz group without pseudozeros is essentially $R^{m}$ with the usual topology and tight order. We show that a locally compact tight Riesz group, $(G, \leqq)$, without pseudozeros, is algebraically and topologically isomorphic with $R^{m} \oplus D$, where $D$ is discrete. $R^{m} \oplus\{0\}$ is a clopen 0 -ideal; and we give necessary and sufficient conditions for $G$ to be isomorphic with $R^{m} \oplus D$ in all respects. Further $(G, \leqq)$ contains an $o$-ideal isomorphic with $R^{m} \oplus \Sigma_{\mathrm{x}} Z$ and $G$ is isomorphic with it if and only if $(G, \leqq, \mathscr{U})$ is interval-compact.

\section{Preliminaries}

Let $(G, \leqq)$ be an abelian pogroup. Denote the set $\{x: x \geqq 0\}$ by $P$ and the set $\{x: x>0\}$ by $P^{*}$. We say $x$ is pseudopositive if $x+p>0$ for all $p>0$ and $x \geq 0$. We say $x$ is a pseudozero if $x$ and $-x$ are pseudopositive. The intervals

$$
(a, b)=\{x: a<x<b\} a, b \in G
$$

form a subbase for the open-interval topology $\mathscr{U}$ on $G$.

If $(G, \leqq)$ is dense then $(G, \mathscr{U})$ is a topological group. Also $(G, \mathscr{U})$ is $T_{1}$ if and only if $(G, \leqq)$ has no pseudozeros. Denote the closure of $S \subseteq G$ in $\mathscr{U}$ by $S^{-}$. We say $(G, \leqq, \mathscr{U})$ is interval-compact Cameron and Miller (to appear) if $(a, b)^{-}$ is compact for every $a<b$.

If $(G, \leqq)$ has no pseudozeros we write $x>0$ to mean that $x>0$ or that $x$ is pseudopositive. Then $(G, \preccurlyeq)$ is a pogroup and $\preccurlyeq$ is called the associated order. We write $a \gg b$ if $a \preccurlyeq n b$ for all integers $n$, and $(G, \leqq)$ is said to be archimedean if $a \gg b$ implies that $b=0$. If $(G, \leqq)$ is an $l$-group, we say $(G, \leqq)$ is a complete $l$-group if each bounded subset of $G$ has a sup and an inf. Every complete $l$-group is archimedean. If, given $a, b>0$ there exists some integer $n$ such that $n a>b$, we say $(G, \leqq)$ is strongly archimedean (Loy and Miller (1972) call this archimedean). A strongly archimedean $l$-group is archimedean. We say $(G, \leqq)$ has the 
$\operatorname{TR}(m, n)$ interpolation property, Cameron and Miller (to appear), if, given $a_{1}, \cdots, a_{m}, b_{1}, \cdots, b_{n} \in G$ such that $a_{i}<b_{j}$ for $i=1,2, \cdots, m$ and $j=1,2, \cdots, n$ there exists $c \in G$ such that $a_{i}<c<b_{j}$ for $i=1, \cdots, m$ and $j=1, \cdots, n$. A tight Riesz group (abbreviated TRG) is a directed abelian pogroup satisfying $\operatorname{TR}(2,2)$. We say a pogroup has small elements if given $a>0$ and $n$, a positive integer, there exists $b>0$ such that $a>n b$. Divisible isolated pogroups and pogroups satisfying $\operatorname{TR}(1,2)$ have small elements.

Let $\left\{G_{i}, \preccurlyeq\right\}_{i \in I}$ be a family of $l$-groups, denote. their (small) direct sum by $\sum G_{i}$ and define $\preccurlyeq$ on $\Sigma G_{i}$ by, $\left(g_{i}\right) \geqslant 0$ if $g_{i} \geqslant 0$ for all $i \in I$. Denote the direct sum of two groups $G_{1}$ and $G_{2}$ by $G_{1} \oplus G_{2}$. If $(G, \preccurlyeq)$ is an $l$-group and $S$ a subset of $G$ denote the set

$$
\{x:|x| \wedge|s|=0 \text { for all } s \in S\}
$$

by $S^{\perp}$. Let $Z, R$ denote the abelian groups of integers and reals. If $x=\left(x_{1}, \cdots, x_{n}\right)$ $\in R^{n}$ we say $x>0$ if $x_{i}>0$ for $i=1, \cdots, n$. We call this the tight order. Then the open-interval topology is precisely the euclidean topology $\mathscr{E}$, and $x \geqslant 0$ if $x_{i} \geqq 0$ for $i=1, \cdots, n$.

\section{Locally compact and interval-compact TRGs}

Let $(G, \leqq)$ be a non-trivially ordered abelian pogroup. We recall two results of Loy and Miller (1972), in fact Theorem 1 is the main result of that paper.

LEMMA 1. (Loy and Miller). Let $(G, \leqq)$ be a locally compact $T R G$ such that $(G, \preccurlyeq)$ is an l-group, then $(G, \mathscr{U})$ is algebraically and topologically isomorphic with $R^{m} \oplus D$, where $m$ is a positive integer and $D$ is discrete.

Proof. This follows immediately from $\S 4,6^{\circ}$ of Loy and Miller (1972) and a theorem of G. W. Mackey's (see Loy and Miller (1972)).

THEOREM 1. (Loy and Miller) Let $(G, \leqq, \mathscr{U})$ be a strongly archimedean locally compact TRG without pseudozeros, then it is isomorphic in all respects with $\left(R^{m}, \leqq, \mathscr{E}\right)$ for some positive integer $m$.

The result of Lemma 1 can be generalized, without changing the method of proof, to the following Lemma.

LEMMA 2. Let $(G, \leqq, \mathscr{U})$ be an isolated locally compact pogroup with small elements and without pseudozeros, then $(G, \mathscr{U})$ is algebraically and topologically isomorphic with $R^{m} \oplus D$, where $m$ is a non-negative integer and $D$ is discrete.

We also give a modification of Theorem 1 in terms of small elements. 
THEOREM 2.Let $(G, \leqq, \mathscr{U})$ be a strongly archimedean locally compact pogroup with small elements such that $(G, \preccurlyeq)$ is an l-group, then it is isomorphic in all respects with $\left(R^{m}, \leqq, \mathscr{E}\right)$ for some positive integer $m$.

Proof. Firstly we show that $(G, \leqq)$ is isolated. If $n x>0$ for some positive integer $n$, then for some $p>0, n x>n p$. So certainly $x \geqslant p$, since $(G, \preccurlyeq)$ is isolated, and so $x>0$. Now we show that $(G, \leqq)$ is TR(1,2). Let $a, b>0$, then for some positive $n, n a>b$ and for some $c>0, b>n c$. So $a, b>c>0$, and hence $(G, \leqq)$ is $\operatorname{TR}(1,2)$. It can easily be shown that if $(G, \leqq)$ is $\operatorname{TR}(1,2)$ and $(G, \preccurlyeq)$ is an $l$-group then in fact $(G, \leqq)$ is $\operatorname{TR}(2,2)$. Clearly $(G, \leqq)$ is directed, so it is a TRG. Now apply Theorem 1 .

If we remove "strongly archimedean" from the hypothesis of Theorem 1, we can still show that at least an $o$-ideal (convex directed subgroup) of $(G, \leqq)$ is isomorphic with $R^{m}$.

THEOREM 3. Let $(G, \leqq, \mathscr{U})$ be an isolated locally compact $T R G$ without pseudozeros, then there exists a clopen o-ideal $H$ and $a$ positive integer $m$ such that:

(i) $(H, \leqq, \mathscr{U})$ is isomorphic in all respects with $\left(R^{m}, \leqq, \mathscr{E}\right)$,

(ii) if $x \in P^{*}$ and $y \in H \cap P^{*}$ then $n x>y$ for some positive integer $n$,

(iii) if $(G, \prec)$ is an l-group then $(H, \preccurlyeq)$ is an l-ideal.

Proof. Let $\theta$ denote the isomorphism indicated in Lemma 2. If $\alpha \in R^{m}$ and $\alpha>0$ then for some $a \in P^{*}$, we have

$$
\theta\{(-a, a)\} \subseteq(-\alpha, \alpha) \oplus\{0\} .
$$

In a TRG $(0, n a)=(0, a)+\cdots+(0, a) .\left(\$ 2,4^{\circ}\right.$ of Loy and Miller (1972)), so $\theta\{(0, n a)\} \subseteq R^{m} \oplus\{0\}$ and $\theta\{(-n a, 0)\} \subseteq R^{m} \oplus\{0\}$. Let

$H=\{x:-n a<x<n a$ for some positive integer $n\}$, then it is easily shown that $\theta(H) \subseteq R^{m} \oplus\{0\}$. Also for some positive integer $k$,

$$
\theta\{(-a, a)\} \supseteq\left(-\frac{\alpha}{k}, \frac{\alpha}{k}\right) \oplus\{0\} .
$$

So $\theta(H)=R^{m} \oplus\{0\}$. It is easily shown that $H$ is an $o$-ideal in $(G, \leqq) . H$ is an open subgroup of a topological group and hence closed. Also the induced topology on $H$ is homeomorphic with the open-interval topology on $(H, \leqq)$, hence $(H, \leqq)$ has no pseudozeros and is locally compact in its open-interval topology.

If $b \in H \cap P^{*}$ then for some $\beta \in R^{m}, \beta>0$, we have

$$
\theta\{(-b, b)\} \supseteq(-\beta, \beta) \oplus\{0\},
$$

so $H=\{x:-n b<x<n b$ for some positive integer $n\}$. Hence $(H, \leqq)$ is strongly archimedean. So by Theorem 1, (i) follows. 
If $x \in P^{*}$ and $y \in H \cap P^{*}$ then there exists $z \in H \cap P^{*}$ such that $x, y>z>0$. By (i) $n z>y$ for some positive $n$, so $n x>y$. The proof of (iii) is trivial.

We show in Theorem 4 that the result of Theorem 3 can be improved; in fact the condition that $(G, \leqq)$ is isolated can be deleted.

If $(G, \leqq)$ is a locally compact TRG such that $(G, \preccurlyeq)$ is an l-group, is the isomorphism referred to in Lemma 1 also an order isomorphism? That is, is $(D, \preccurlyeq)$ an $l$-group such that, if $a \in G$ and $\theta(a)=\langle\alpha, d\rangle$ we have $a>0$ if and only if $\alpha>0$ and $d \geqslant 0$, and $a \geqslant 0$ if and only if $\alpha \geqslant 0$ and $d \geqslant 0$ ?

LEMMA 3. Let $(G, \preccurlyeq)$ be an l-group with an l-ideal $(H, \preccurlyeq)$ isomorphic with $\left(R^{m}, \preccurlyeq\right)$ for some positive integer $m$. Then $G \equiv H \oplus H^{\perp}$ if and only if there does not exist $x \in G$ and $h \in H, h \succ 0$ such that $x \gg h$.

Proof. Certainly the condition is necessary. Now suppose that $x \gg h$ is false for all $x \in G$ and all $h \in H, h \succ 0$. Let $a \in G, a \succ 0$, and consider the set $\{a \wedge h: h \in H\}$. This is a subset of $H$ and so it is bounded above in $H$ since otherwise, $a \gg e_{i}$, for some $i$, where $e_{i}=(0,0, \cdots, 0,1,0, \cdots), e_{i} \in R^{m} \equiv H$. Hence $\vee\{a \wedge h: h \in H\}$ exists for all $a>0$, and belongs to $H$. It follows that $G \equiv H \oplus H^{\downarrow}$ (see proof of Theorem 16, Fuchs (1963; page 91)).

Corollary. If $(G, \leqq)$ is a locally compact $T R G$ such that $(G, \preccurlyeq)$ is an l-group, then $G$ is isomorphic in all respects with $R^{m} \oplus D$, if and only if there does not exist an integer $i$ and $x \in G$ with $x \gg e_{i}$.

Denote the set $\left\{x \geqq 0 ;(0, x)^{-}\right.$is compact $\}$by $C$. It is a consequence of a result in Cameron and Miller (to appear) that if $(G, \leqq)$ is an interval-compact TRG without pseudozeros, then $(G, \preccurlyeq)$ is a complete $l$-group. Below we characterize all such groups completely.

THEOREM 4. Let $(G, \leqq, \mathscr{U})$ be a locally compact $T R G$ without pseudozeros, then:

(i) $C$ is a convex subsemigroup of $(P, \leqq)$,

(ii) the subgroup $(C-C$, () , generated by $C$, is an l-group isomorphic in all respects with $R^{m} \oplus \Sigma_{\mathbb{N}} Z$, for some positive integer $m$ and cardinal $\aleph$,

(iii) the condition in Theorem 3 that $(G, \leqq)$ is isolated is redundant.

Proof. (i) It is obvious that $C$ is convex. In $\S 2,14^{\circ}$ Loy and Miller (1972) it is shown that $C$ is a subsemigroup of $P$.

(ii) It follows easily that $(C-C, \leqq)$ is interval-compact in its open-interval topology. Also $(C-C, \leqq)$ is a TRG without pseudozeros, so $(C-C, \preccurlyeq)$ is a complete $l$-group. We now apply Lemma 3 Corollary. So $C-C$ is isomorphic in all respects with $R^{m} \oplus D_{1}$, for some discrete l-group $\left(D_{1}, \preccurlyeq\right)$. Further the strictly positive cone of $\left(D_{1}, \preccurlyeq\right)$ can have no infinite descending chains. For 
suppose that $d_{1} \succ d_{2} \succ \cdots \succ 0, d_{i} \in D_{1}$, and let $\left.\alpha \in R^{m}, \alpha\right\rangle 0$, and $a=\theta^{-1}\left\langle\alpha, d_{1}\right\rangle$. By Lemma 3 Corollary $a>0$ so $(0, a)^{-}$is compact. Let $b=\theta^{-1}\langle\alpha, 0\rangle$, and cover $(0, a)^{-}$by sets $(x-b, x+b)$ where $x \in(0, a)^{-1}$. Then clearly $\theta^{-1}\left\langle 0, d_{i}\right\rangle$ all belong to different covers, contradicting the compactness of $(0, a)^{-}$. It follows from Birkhoff $(1967$; page 299$)$ that $\left(D_{1}, \preccurlyeq\right)$ is isomorphic with $\left(\Sigma_{k} Z, \preccurlyeq\right)$ for some cardinal $\aleph$.

(iii) Write $(H, \leqq)$ for $\left(R^{m}, \leqq\right)$ in (ii).

CoROLLARY. Let $(G, \leqq, \mathscr{U})$ be an interval-compact $T R G$, then $G$ is isomorphic in all respects with $R^{m} \oplus \Sigma_{\star} Z$, for some positive integer $m$ and some cardinal $\aleph$, and conversely.

\section{Acknowledgements}

This research was supported by a Commonwealth Postgraduate Award. The author is grateful to Professor J. B. Miller for his helpful advice.

\section{References}

G. Birkhoff (1967), Lattice theory (Amer. Math. Soc., Providence, 1967, 3rd. ed.).

N. Cameron and J. B. Miller (to appear), 'Topology and axioms of interpolation in partially ordered spaces', J. Reine Angew. Math.

L. Fuchs (1963), Partially ordered algebraic systems (Pergamon, Oxford, 1963).

R. J. Loy and J. B. Miller (1972), 'Tight Riesz groups', J. Austral. Math. Soc. 13, 224-240.

Department of Mathematics

Monash University

Clayton

Victoria 3168

Australia 